\title{
A Long-term Response to Nivolumab in a Case of PD-L1-negative Lung Adenocarcinoma with an EGFR Mutation and Surrounding PD-L1-positive Tumor-associated Macrophages
}

\author{
Hiromi Watanabe ${ }^{1,2}$, Kadoaki Ohashi ${ }^{2}$, Kazuya Nishii ${ }^{1}$, Keisuke Seike ${ }^{1}$, Go Makimoto ${ }^{1}$,
} Katsuyuki Hotta ${ }^{2,3}$, Yoshinobu Maeda ${ }^{1}$ and Katsuyuki Kiura ${ }^{2}$

\begin{abstract}
:
Anti-programmed cell death 1 (PD-1) antibodies have poor efficacy in epidermal growth factor receptor $(E G F R)$-mutated lung cancer. We herein report a 72-year-old man with programmed cell death-ligand 1 (PDL1)-negative lung adenocarcinoma harboring an EGFR mutation that responded to nivolumab for more than 2 years. A pathological examination revealed infiltration of CD8-positive lymphocytes and macrophages expressing CD68, CD206, and PD-L1 into the PD-L1-negative tumor; CD206 expression is a marker of immunosuppressive tumor-associated macrophages (TAMs). The presence of PD-L1-positive TAMs in the tumor environment might be a predictor of a positive response to anti-PD-1 antibodies.
\end{abstract}

Key words: lung adenocarcinoma, EGFR mutations, nivolumab, tumor-associated macrophages

(Intern Med 58: 3033-3037, 2019)

(DOI: 10.2169/internalmedicine.2875-19)

\section{Introduction}

Immune checkpoint inhibitors (ICIs) have fundamentally changed the lung cancer treatment strategy. However, antiprogrammed cell death 1 (PD-1) antibodies have poor efficacy in epidermal growth factor receptor $(E G F R)$-mutated lung cancer (1-3). Possible explanations for the poor efficacy are a low tumor mutation burden (TMB), low expression of programmed cell death-ligand 1 (PD-L1), or low immunogenicity, although the precise mechanisms remain to be clarified.

We herein report a case of PD-L1-negative lung adenocarcinoma harboring an EGFR mutation that responded to nivolumab for more than two years.

\section{Case Report}

A 72-year-old man who was a former smoker (48 pack- years) was diagnosed with locally advanced lung adenocarcinoma (cT1bN2M0, cStage IIIA) harboring an EGFR exon 19 deletion. He underwent right upper lobectomy after neoadjuvant chemoradiotherapy with cisplatin plus docetaxel and 46 Gy of thoracic radiation. Twelve months after the surgery, brain magnetic resonance imaging indicated recurrence of multiple brain metastases; subsequently, the brain lesions were treated with $\gamma$-knife therapy. Thirteen months after radiosurgery, a computed tomography (CT) scan revealed multiple lung metastases.

Salvage chemotherapies, including EGFR tyrosine kinase inhibitors (TKIs), erlotinib, carboplatin plus paclitaxel, and docetaxel, were then administered consecutively. Erlotinib as second-line therapy was continued for seven months with a partial response. After docetaxel therapy, erlotinib was readministered and generated a response for four months, but eventually, multiple lung metastatic lesions regrew. At the time of progression, osimertinib was not approved for clinical practice; therefore, the patient did not agree to a re-

\footnotetext{
${ }^{1}$ Department of Hematology, Oncology and Respiratory Medicine, Okayama University Graduate School of Medicine, Dentistry and Pharmaceutical Sciences, Japan, ${ }^{2}$ Department of Respiratory Medicine, Okayama University Hospital, Japan and ${ }^{3}$ Center for Innovative Clinical Medicine, Okayama University Hospital, Japan

Received: February 17, 2019; Accepted: May 13, 2019; Advance Publication by J-STAGE: July 10, 2019

Correspondence to Dr. Kadoaki Ohashi, kohashi@cc.okayama-u.ac.jp
} 
HE
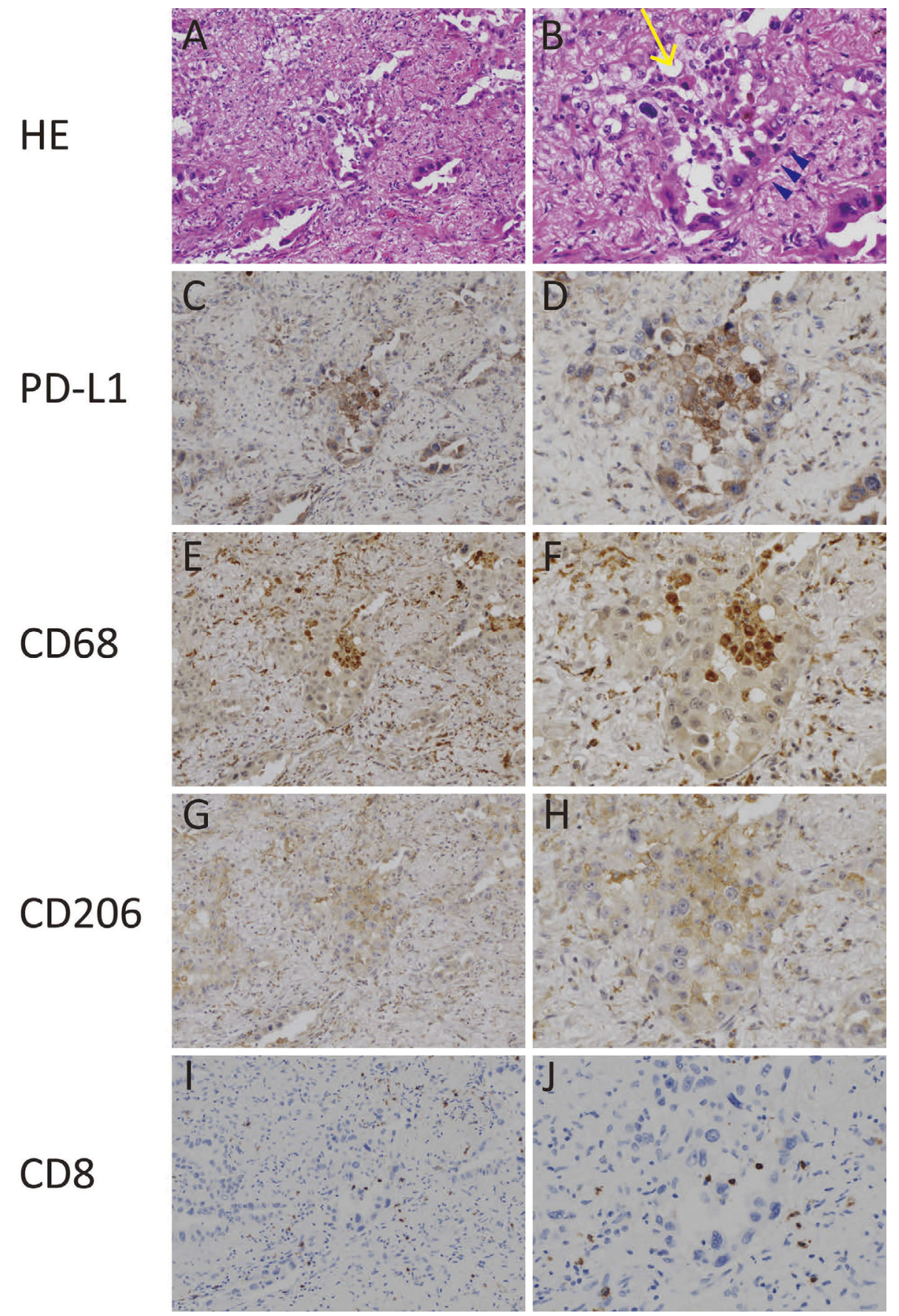

Figure 1. PD-L1-positive M2 macrophages surrounding PD-L1-negative tumors. Microscope magnification: (A, C, E, G, I: 200-fold; B, D, F, H, J: 400-fold). Anti-PD-L1 antibody (28-8) (Dako SK005), anti-CD68 antibody (Abcam Cat\# ab955), anti-CD206 antibody (Abcam Cat\# ab64693) and antiCD-8 antibody (Roche Cat\# 7904460) were used for immunostaining. A, B: Hematoxylin and Eosin staining. The blue arrowhead indicates lung cancer cells. The yellow arrow indicates macrophages. $\mathrm{C}$, D: The tumor did not express PD-L1. Instead, the surrounding cells showed high PD-L1 expression. E, F: The PD-L1-positive cells were CD68-positive macrophages. G, H: CD206 staining was also positive in the macrophages. I, J: CD8-positive lymphocytes were observed in the vicinity of the cancer cells.

biopsy for testing of his EGFR mutation status. Although the PD-L1 expression result for the surgical specimen tumors was negative (Fig. 1A-D), nivolumab was administered as sixth-line therapy. After seven cycles of nivolumab administration, CT revealed that the metastatic lung tumors and the mediastinal and subclavian lymph node tumors had almost disappeared. Currently, he has continued treatment with nivolumab for more than two years (Fig. 2). Thus far, there has been no evidence of tumor regrowth or serious immune-related adverse events. 


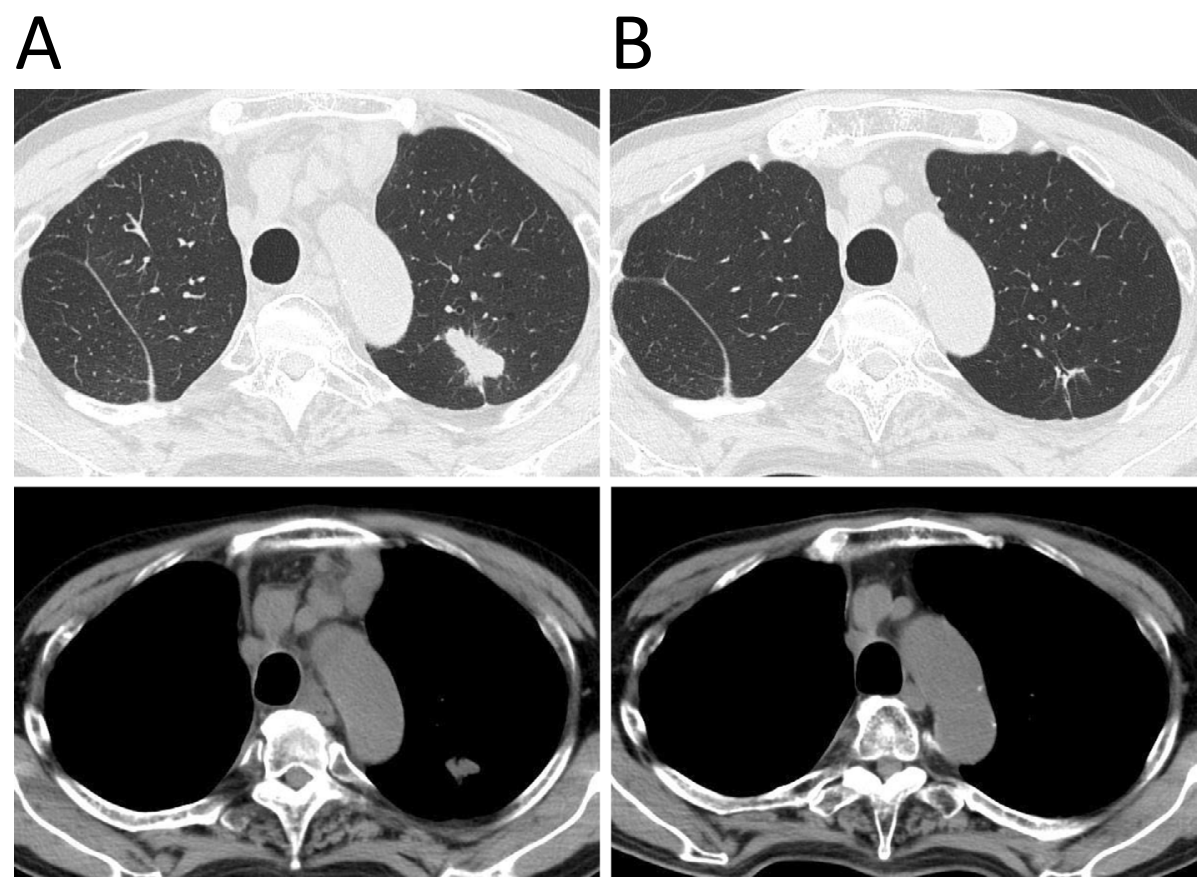

Figure 2. Chest computed tomography (CT) of the metastatic lung tumors and mediastinal and subclavian lymph node tumors. A: Chest CT scan at the beginning of nivolumab treatment. B: Chest CT scan two years after starting nivolumab treatment.

A further pathological examination revealed CD8-positive lymphocytes and macrophages expressing CD68 and PD-L1 that infiltrated the PD-L 1-negative tumor (Fig. 1E-F and 1I-J). Some of the CD68-positive macrophages were also positive for CD206, which is a marker of immunosuppressive tumor-associated macrophages (TAMs) (Fig. 1G-H, 3).

\section{Discussion}

Nivolumab was found to produce a long-term response in lung adenocarcinoma harboring EGFR exon 19 deletion. The lung tumors did not express PD-L1 but were surrounded with CD8-positive T-lymphocytes and immune suppressive TAMs expressing PD-L1. At present, no marker has been established for predicting a positive effect of anti-PD-1 antibody in lung cancer harboring EGFR mutations; however, our case suggested that the presence of PD-L1-positive TAMs might predict positive effects of nivolumab.

EGFR-TKIs are a standard treatment option for lung cancer with EGFR mutations. Osimertinib has been approved for EGFR-TKI naïve lung tumors; therefore, the role of EGFR-TKIs is more important than ever. However, unlike ICIs, EGFR-TKIs do not result in deep remission in advanced lung cancers. Unfortunately, a recent clinical trial showed that the anti-PD-1 antibody pembrolizumab had a limited effect on EGFR-TKI-naïve lung cancers harboring EGFR mutations, even if the tumors had a high PD-L1 expression (4). The treatment sequence might be one of the influencing factors; however, there is thus far no evidence that prior therapy influences the treatment effect of nivolumab $(1,5)$.

A recent report showed that a Brinkman index $\geq 600$, duration of EGFR-TKI administration < six months, and uncommon EGFR mutations were correlated with a longer progression-free survival with nivolumab in EGFR-mutant lung cancers (6). Our patient was a heavy smoker, but the tumor harbored a common activating EGFR mutation and responded well to EGFR-TKI for more than six months.

The tumor microenvironment may also influence the effects of ICI treatment (7). A recent report showed that large numbers of CD8-positive lymphocytes and small numbers of forkhead box protein 3 (FOXP3)-positive tumor-infiltratinglymphocytes were related to the effect of nivolumab in EGFR-mutant lung cancer that developed resistance to EGFR-TKIs (8). The presence of immune suppressive M2 macrophages in the tumor microenvironment has been reported to be correlated with a poor prognosis of lung cancer $(9,10)$. Another study showed that macrophages impede CD8 T-cells from reaching tumor cells and limit the efficacy of anti-PD-1 treatment against lung squamous cell cancers in humans and mice models (11). Furthermore, the presence of PD-L1-positive tumor-infiltrating immune cells, including macrophages, showed a correlation with positive effects of anti-PD-L1 antibody in clinical trials involving lung cancers (12). The positive correlation between the effect of antiPD-1 antibody and the presence of PD-L1-positive macrophages in the tumor microenvironment has also been reported in melanoma $(13,14)$. Taken together, these data may suggest that immune suppressive TAMs expressing PD-L1 negatively affect cytotoxic lymphocytes, and this negative interaction may be a potential therapeutic target of 


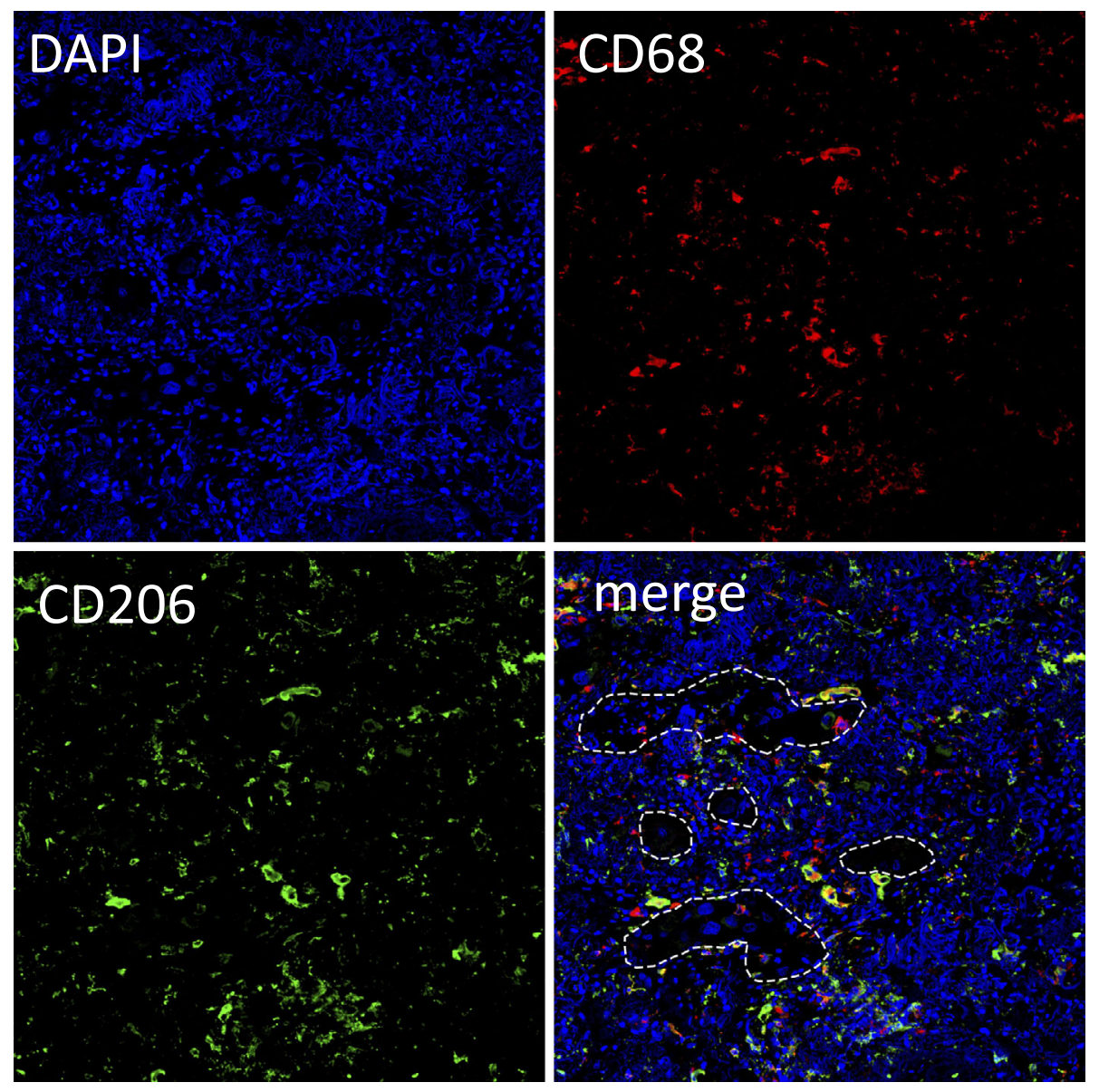

Figure 3. M2 macrophages surrounding the lung tumors. Double staining with anti-CD68 antibody (Abcam Cat\# ab955) and anti-CD206 antibody (Abcam Cat\# ab64693) was performed. The antiCD68 antibody appeared red due to the secondary fluorescent conjugated antibody (Alexa Fluor ${ }^{\circledR} 594$ Abcam ab150116). The anti-206 antibody appeared green due to the secondary fluorescent conjugated antibody (Alexa Fluor ${ }^{\circledR} 488$ Abcam ab150077). Images were overlaid to demonstrate co-localization. Blue: DAPI, Red: CD68, Green: CD206. The white dotted line indicates tumor cells.

\section{ICIs (15).}

At present, macrophages are thought to be a promising therapeutic target $(16,17)$. One strategy involves the direct inhibition of immunosuppressive TAMs $(11,16)$, while another involves inducing polarization from immunosuppressive M2 phenotype to immune stimulative M1 phenotype $(18,19)$. Recent studies have shown that anti-PD-1 or anti-PD-L1 antibodies activate the ability of macrophages on phagocytosis to promote polarization to the M1 phenotype (20-22). Therefore, the immune activation of macrophages by anti-PD-1 or PD-L1 antibody might be involved in patients with PD-L1-negative tumors showing unexpected response to ICIs.

In our case, a pathological examination revealed CD8positive lymphocytes and macrophages expressing CD68 and PD-L1, which infiltrated the PD-L1-negative tumor (Fig. 1E-F and I-J). The CD68-positive macrophages were also CD206-positive, which is a marker of immunosuppressive TAMs (Fig. 1G-H). Therefore, we speculate that the following scenarios might explain the mechanisms underlying the excellent therapeutic effect in our case: 1) TAMs suppressed CD8-positive lymphocytes, 2) nivolumab inhibited the negative interaction of M2 macrophages, and 3) CD 8-positive lymphocytes were released and attacked the tumors. Alternatively, nivolumab might promote polarization from the M2 phenotype to the M1 phenotype, at which point the M1 macrophages suppress the tumors.

In addition to the PD-L1 status of tumors, the presence of PD-L1-positive TAMs in the tumor environment might be a predictive biomarker of a positive response to anti-PD-1 antibodies. Further studies are warranted to identify clues about which patients would benefit from ICIs for lung cancers harboring EGFR mutations.

The authors state that they have no Conflict of Interest (COI).

\section{Financial Support}

This work was supported by JSPS Grants-in-Aid for Scientific Research [Grant-in-Aid for Young Scientists (B): KAKEN 16K 19454] to K.O. and JSPS Grants-in-Aid for Scientific Research [Scientific Research (B): KAKEN 15H04830] to K.O. and K.K. 


\section{Acknowledgement}

First, we thank the patient and his family. We are grateful to Hiromi Nakashima and Kyoko Maeda for the technical support. We are also grateful to Hirofumi Inoue, Department of Pathology Okayama University Hospital, for his support in the pathological studies.

\section{References}

1. Gainor JF, Shaw AT, Sequist LV, et al. EGFR mutations and ALK rearrangements are associated with low response rates to PD-1 pathway blockade in non-small cell lung cancer: a retrospective analysis. Clin Cancer Res 22: 4585-4593, 2016.

2. Lee CK, Man J, Lord S, et al. Checkpoint inhibitors in metastatic EGFR-mutated non-small cell lung cancer-a meta-analysis. J Thorac Oncol 12: 403-407, 2017.

3. Lee CK, Man J, Lord S, et al. Clinical and molecular characteristics associated with survival among patients treated with checkpoint inhibitors for advanced non-small cell lung carcinoma: a systematic review and meta-analysis. JAMA Oncol 4: 210-216, 2018.

4. Lisberg A, Cummings A, Goldman JW, et al. A phase II study of pembrolizumab in EGFR-mutant, PD-L1+, tyrosine kinase inhibitor (TKI) naïve patients with advanced NSCLC. J Thorac Oncol 13: 1138-1145, 2018.

5. Kataoka Y, Ebi N, Fujimoto D, et al. Prior radiotherapy does not predict nivolumab response in non-small-cell lung cancer: a retrospective cohort study. Ann Oncol 28: 1402, 2017.

6. Yoshida H, Kim YH, Ozasa H, et al. Nivolumab in non-small-cell lung cancer with EGFR mutation. Ann Oncol 29: 777-778, 2018.

7. Pitt JM, Marabelle A, Eggermont A, Soria JC, Kroemer G, Zitvogel L. Targeting the tumor microenvironment: removing obstruction to anticancer immune responses and immunotherapy. Ann Oncol 27: 1482-1492, 2016.

8. Haratani K, Hayashi H, Tanaka T, et al. Tumor immune microenvironment and nivolumab efficacy in EGFR mutation-positive nonsmall-cell lung cancer based on T790M status after disease progression during EGFR-TKI treatment. Ann Oncol 28: 1532-1539, 2017.

9. Mei J, Xiao Z, Guo G, et al. Prognostic impact of tumorassociated macrophage infiltration in non-small cell lung cancer: A systemic review and meta-analysis. Oncotarget 7: 34217-34228, 2016.

10. Wu P, Wu D, Zhao L, et al. Inverse role of distinct subsets and distribution of macrophage in lung cancer prognosis: a meta- analysis. Oncotarget 28: 41451-40460, 2016.

11. Paranzoni E, Lemoine J, Vimeux L, et al. Macrophages impede CD8 $\mathrm{T}$ cells from reaching tumor cells and limit the efficacy of anti-PD-1 treatment. PNAS 115: E4041-E4050, 2018.

12. Herbst RS, Soria JC, Kowanetz M, et al. Predictive correlates of response to the anti-PD-L1 antibody MPDL3280A in cancer patients. Nature 515: 563-567, 2014.

13. Tumeh PC, Harview CL, Yearly JH, et al. PD-1 blockade induces responses by inhibiting adaptive immune resistance. Nature 515: 568-571, 2014.

14. Vilain RE, Menzies AM, Willmott JS, et al. Dynamic changes in PD-L1 expression and immune infiltrates early during treatment predict response to PD-1 blockade in melanoma. Clin Cancer Res 23: 5024-5033, 2017.

15. Biswas SK, and Mantovani. A macrophage plasticity and interaction with lymphocyte subsets: cancer as a paradigm. Nat Immunol 11: 889-896, 2010.

16. Brown JM, Recht L, Strober S. The promise of targeting macrophages in cancer therapy. Clin Cancer Res 23: 3241-3250, 2017.

17. Komohara Y, Jinushi M, Takeya M. Clinical significance of macrophage heterogeneity in human malignant tumors. Cancer Sci 105: 1-8, 2014.

18. Mantovani A, Sozzani S, Locati M, Allavena P, Sica A. Macrophage polarization: tumor-associated macrophages as a paradigm for polarized M2 mononuclear phagocytes. Trends Immunol 23: 549-555, 2002.

19. Rolny C, Mazzone M, Tugues S, et al. HRG inhibits tumor growth and metastasis by inducing macrophage polarization and vessel normalization through downregulation of PIGF. Cancer Cell 19: 31-44, 2011.

20. Gordon SR, Maute RL, Dulken BW, et al. PD-1 expression by tumour-associated macrophages inhibits phagocytosis and tumour immunity. Nature 545: 495-499, 2017.

21. Kuang DM, Zhao Q, Peng C, et al. Activated monocytes in peritumoral stroma of hepatocellular carcinoma foster immune privilege and disease progression through PD-L1. J Exp Med 206: 13271337, 2009.

22. Mediavilla-Varela M, Page MM, Kreahling J, Antonia SJ, Altiok S. Anti-PD1 treatment to induce M1 polarization of tumor infiltrating macrophages in a $3 \mathrm{D}$ ex vivo system of lung cancer patients. J Clin Oncol 35 (15_Suppl): e23090, 2017.

The Internal Medicine is an Open Access journal distributed under the Creative Commons Attribution-NonCommercial-NoDerivatives 4.0 International License. To view the details of this license, please visit (https://creativecommons.org/licenses/ by-nc-nd/4.0/).

(C) 2019 The Japanese Society of Internal Medicine Intern Med 58: 3033-3037, 2019 